\title{
DISTRIBUTED SIMULATION IN INDUSTRY - A SURVEY PART 2 - EXPERTS ON DISTRIBUTED SIMULATION
}

\author{
Csaba Attila Boer \\ TBA BV \\ Vulcanusweg 259, \\ 2624 AV, Delft, \\ THE NETHERLANDS
}

\author{
Arie de Bruin \\ Delft University of Technology \\ Faculty of Electrical Engineering, \\ Mathematics and Computer Science \\ Mekelweg 4, 2628 CD, Delft \\ THE NETHERLANDS
}

\author{
Alexander Verbraeck \\ Delft University of Technology \\ Faculty of Technology, Policy and \\ Management \\ Jaffalaan 5, 2628 BX, Delft \\ THE NETHERLANDS
}

\begin{abstract}
Distributed simulation is used very little in industry, especially when compared with the interest in distributed simulation from research and from the military domain. In order to answer the question why industry lags behind, the authors have carried out an extensive survey, using a questionnaire and interviews, with users, vendors, and developers of distributed simulation products, as well as with vendors of non-distributed simulation software. This paper reports on the second part of the survey, namely a series of open ended interviews. We report on the responses we obtained indicating the discrepancies between the different "worlds". A categorization of these responses is given using which it is possible to formulate clear guidelines for further developments of standards for distributed simulation.
\end{abstract}

\section{INTRODUCTION}

In our companion paper (Boer, et al. 2006a), we discussed our observation, shared with many others, that distributed simulation, and HLA, is hardly used in industry whereas it is frequently applied in the defense domain. This observation has inspired us to set up a research program in which we study three research questions. We try to find out whether our observation is really true, what the reasons behind this phenomenon might be, and what actions can be undertaken to remedy this situation.

Our research plan was to first perform an extensive survey in the field in which we solicited experts on (distributed) simulation and HLA to share their views. The survey part was set up along the lines of the Delphi methodology (Linstone and Turoff 1975), (Linstone 1978), which we briefly discuss in (Boer, et al. 2006a) as well. This methodology defines an iterative process in which results from earlier surveys are used as the basis for more extensive and better focused new ones.
The first step of our survey was a questionnaire targeted at the vendors of COTS (Commercial-Off-The-Shelf) simulation packages which are widely used in industry. This survey mostly focused on the question whether it is true that the HLA standard is hardly used in industry. Although we did not obtain a conclusive answer, the survey provided a sufficient amount of evidence in favor of our observation. As a side effect we obtained some hints regarding the other aforementioned research questions as well. We report on this questionnaire survey in (Boer, et al. 2006a).

On the basis of these results we then performed an interview survey. Besides the COTS vendors we selected people that we consider to be experts in distributed simulation who have already had experience with distributed simulation either in industry or defense. The setup of our survey has been depicted as Figure 1 in (Boer, et al. 2006a).

We thus applied two types of data collection: firstly a structured questionnaire for COTS simulation package vendors, and secondly an interview with open ended questions based on our observations and the results of the questionnaire. The interview aims to focus more deeply on the research questions. As we have stated before, the interview is based to some extent on the questionnaire and accordingly, it can be considered as a second iteration in this research. For the interview we employed purposeful selection in order to determine the participants. The data in this case has been collected through a phone interview with open ended questions. In order to analyze the data collected from the interviews we applied qualitative content analysis.

Data analysis in qualitative research involves preparing the data for analysis, conducting different analyses, moving deeper and deeper into understanding the data, representing the data, and making a global interpretation of the data (Creswell 2003), (Seale, et al. 2004). Several generic processes are described in the literature to convey the 


\section{Boer, De Bruin, and Verbraeck}

overall activities of qualitative data analysis. In this research we follow the generic steps presented in (Creswell 2003, pg. 191-195).

- Organize and prepare the data for analysis.

- Read through all data.

- Begin detailed analysis with a coding process.

- Use a coding process to generate a detailed description of topics and subtopics.

- The final step in data analysis involves making an interpretation or meaning of the data.

This paper is organized as follows. In the next section we describe the way the interview is set up, viz. selection of the participants, the design of the interview and the method used for collecting the data. Section 3 is devoted to an overview of the answers we obtained. In order to apply these results and to work towards a solution of the problem that distributed simulation is hardly applied in industry, we need to perform an analysis on this data. This is described in Section 4. Finally Section 5 concludes.

The outcome of Section 4 is a structured list of topics and subtopics raised during the interviews. This list forms the basis on which we have developed guidelines for further development of standards for distributed simulation in industry. These guidelines have taken the form of a requirements list for an industrial COTS based distributed simulation architecture. We report on this in (Boer, et al. 2006b).

\section{THE INTERVIEW}

This section includes three parts. In the first part we describe the way we selected the participants and the groups in which they are classified. Then, in the second part we present the design of the interview. Finally, we describe the method that we have used for collecting the data.

\subsection{Selection of the Participants}

As we mentioned before, through the interviews we aimed to collect data from experts. The interview we conducted involves unstructured and generally open-ended questions intending to elicit views and opinions of the participants.

"The idea behind qualitative research is to purposefully select participants or sites (or documents or visual material) that will best help the researcher understand the problem and the research question. This does not necessarily suggest random sampling or selection of a large number of participants and sites, as typically found in quantitative research" (Creswell 2003, pg. 185). Consequently for the interview we try to select purposefully those experts who, we believe, can support our research with their experience, view and opinions. This leads into questions around how to select experts, and finding criteria on who can be consid- ered to be an expert. An expert is a person whose knowledge in a specific domain, in our case distributed simulation, is obtained gradually through a period of learning and experience (Turban 1995). We chose to select a heterogeneous group of experts, the advantage of which over a homogeneous group being that different groups can sense issues differently, they might consider the problem from a different angle (Schreiber, et al. 2000).

Although is it impossible to provide a definite list of criteria for expert selection, based on the above observations we lay out the main decisive factors according to which we selected the experts:

- The person's knowledge and experience with distributed simulation;

- The circumstances in which the person gained his/her experience: e.g., theoretical or practical circumstances;

- The selected group should be heterogeneous including people both from the industry and the defense simulation community (especially because HLA was designed and developed by defense).

First of all we considered a subset of the COTS vendors, we chose them based on the result of the questionnaire. This group consisted of 2 persons.

The second group consisted of 8 industrial oriented simulation practitioners both from the scientific area and from practice. As a starting point for selecting these experts we considered the HLA-CSPIF group (recently become CSPI Product Development group), which aims to stimulate the applicability of distributed simulation in the industrial domain. We also made use of some pointers to interesting people as provided in the questionnaire by the COTS vendors.

For the defense community, we identified three groups: experts who designed and developed HLA (2 persons), simulation practitioners who apply HLA in defense oriented simulation projects (also 2 persons, for these two groups we considered DMSO, MITRE, TNO-FEL and SISO as starting points), and 2 HLA commercial vendors which were chosen from the DMSO vendor list published on the DMSO website. In this way we tried to avoid bias against HLA, given that we mainly address practitioners who have applied HLA.

\subsection{Design of the Interview}

We designed the interview and collected data along nine main topics:

1. The experience and knowledge of the interviewed experts in the field of distributed simulation, the HLA standard, COTS simulation packages, and general programming languages. This information is espe- 
cially important in order to determine and validate the weight of the participants' opinion.

2. Differences between industrial and defense simulation. The questions around this topic were included in order to evaluate the observation that defense simulation communities mainly use general purpose languages, such as Java, C++, FORTRAN, and so on, for creating simulation models, while the industrial simulation communities use COTS simulation packages. Furthermore, we tried to uncover other differences between these two communities that the experts might have observed. This topic primarily aims to find reasons why distributed simulation gathered ground in defense and hardly appears in industry.

3. Reusability of existing simulation models. As in our view reusability of already existing models seems to be one of the most beneficial motivations for applying distributed simulation, we enquired the participants about their experience concerning reusability. We especially focused on reusability in the context of HLA, namely on reusability of HLA compliant simulation models. Difficulties and efforts invested for reusing models compared with re-implementing the whole model are investigated here. The aim of this topic is to help us to gather motivation for applying distributed simulation, and further to suggest solutions that can filter out the difficulties encountered.

4. Information hiding within the simulation model. Besides reusability information hiding seems to be another motivation for distributed simulation. We tried to elicit information on whether this is indeed the case.

5. Difficulties and benefits of applying the HLA standard. From the questionnaire and also from literature opinions can be heard about the difficulties of using HLA. We tried to discover whether this is a significant reason why HLA in particular and distributed simulation in general does not gain ground in industry.

6. Integrating simulation models developed in COTS simulation packages using the HLA standard. Similarly to the previous section but focusing more on COTS simulation packages, this section intends to investigate whether this is another significant reason why the HLA standard is not considered in the industrial domain.

7. Benefits and disadvantages of integrating simulation models designed and developed in COTS simulation packages. This issue was only discussed with the COTS simulation package vendors, who provide tools to the simulation practitioners. As we aimed at a solution for the integration of different COTS models we were curious about their vision regarding the integration of different COTS simulation packages.
8. Support of commercial HLA tools for the industrial domain. This topic was discussed only with the HLA vendors, who provide commercially available distributed simulation architectures for integrating simulation models mostly for the defense community. We intended to investigate their role in industry and their support for industrial domain.

9. Future visions on "idealized" distributed simulation architectures for the industry. In this part we tried to investigate the vision of experts on distributed simulation from different groups. We posed the question: "How would you imagine an "idealized" distributed simulation architecture for the industrial domain where the COTS simulation packages are frequently applied?". From the answers we aimed to compose a list of requirements for future solutions for distributed simulation architectures.

\subsection{Data Collection}

In order to interview experts there are different options, such as a face-to-face interview, a phone interview or a group interview in which researcher interviews participants in a group (Creswell 2003). In our case, we conducted a phone interview with all participants, except for one who validated the first version of the findings by means of a face to face interview.

The average time for an interview was 58 minutes. Regarding the evaluation of the interview, as a last question, we asked the experts' opinion about it. They were all quite positive both concerning the topic and the content.

\section{THE RESULTS}

Next we summarize the results of the interview organized for each selected group separately. For a more extensive exposition we refer to (Boer 2005).

\subsection{COTS Simulation Package Vendors}

As it stands now, the COTS vendors do not see direct benefits in using distributed simulation to integrate models written using their packages. They see no economic benefit to cooperate with other vendors, and they argue that combining models written in one package can better be achieved in a monolithic way.

Applying distributed simulation is also hampered by the way models are built in industry. Industry "wants results as fast and as cheaply as possible", most models are of a "throw away" type, and they are hardly maintained during their life cycle. COTS vendors also mention the problems around mapping objects from different models and they state that most models are too specific to be reused. Applying HLA is perceived to only generate more problems, because there are no experienced users and HLA 


\section{Boer, De Bruin, and Verbraeck}

is too complex. According to these experts the adaptors available today do not offer a solution to this problem because still too much knowledge is called for to apply this technique. There exist some experience on distributed simulation in industry though, but this focused on reuse at the component level instead of full model reuse.

On the other hand they recognize that combining COTS models using distributed simulation might be fruitful in collaborative design and development, in emulation, in combining simulation with other applications, like ERP, with specialized simulation tools, or in trying to generate speedup through parallelization.

They believe that a good architecture might generate a technology push. The architecture should be simple, offering only minimal functionality, viz. data representation and exchange and time synchronization. Furthermore, integration should be relatively painless and efficient, leading to perspicuous models and clear communication between them.

\subsection{Industrial Simulation Practitioners}

A first relevant observation that was made by several industrial simulation practitioners is that there is a need to separate issues around HLA and COTS from problems that are actually rooted on a higher level, viz. distributed simulation in general.

The experts saw a clear difference in scope between the projects in defense and in industry. Industry is mainly interested in generating direct results, money is the big factor here. Projects are smaller than in defense and in general the models are not reused. COTS packages are designed for such applications. Defense has other objectives, safety and secrecy of the models are relevant, the models are sophisticated, stable and robust. Verification and validation is important, and mostly general purpose programming languages are used.

The industrial simulation practitioners mentioned several drawbacks of the HLA standard. One of these drawbacks is the performance of the current implementations. Experts relate this to the fact that HLA offers too much irrelevant functionality for industry. Minimal functionality for industry would be only time synchronization and entity passing, possibly augmented with the possibility to join and resign from a simulation run and to publish and subscribe to objects. Other problems that were mentioned by practitioners were incompatibility of RTI's and some ambiguities in the HLA specification. Furthermore, the fact that ownership is a notion that is not implemented in COTS packages causes additional problems when one intends to apply this HLA feature. In addition the mapping problem is mentioned by the industrial simulation practitioners as well. This might be solved by defining standard object descriptions. In this process, however, COTS vendors should be involved. Finally there is the issue of cost. There is a trend towards low priced software packages. Adding the cost of integrating or interfacing to an RTI will increase cost and the customers, as it stands now, are not willing to pay more than $10 \%$ for this. On the other hand a remark was made that, given the prices of COTS packages and RTI's, this is attainable.

Several examples from industry are mentioned which have benefited or might benefit from distributed simulation, such as the virtual factory, material flow between two factories, and combining models of production systems with a model of a supply chain. These are typical applications where cooperation is of benefit to all partners involved. A problem here is that currently there do not exist proper tools to solve the problems involved. It is expected that in the future these types of projects will gain importance, and additionally further globalization will generate a need for more cooperation. The industrial simulation practitioners perceive that distributed simulation has a place in industry, however, industry needs to be convinced on the benefits of distributed simulation. In order to realize this, time and realistic cases are needed.

A big hindrance is that distributed simulation and HLA are complex phenomena of a technical nature, whereas the practitioners in industry are generally not technically inclined. Although HLA takes care of quite some low level technicalities its interface is still too complex for industry. On the question whether adaptors and middleware solve this problem the opinions diverge. Even if HLA is integrated into the modeling paradigm of COTS packages, the user is still exposed to the HLA functionality. Using stand alone adaptors, that would also avoid being tied to a single RTI implementation, might give some relief here according to some experts. Others suggest to aim at an architecture that gives an interface much like a stand alone COTS implementation using which one can specify at run time whether and how the model should be distributed.

\subsection{HLA Designers and Developers}

The experts provided insightful comments on the difference between simulation models built by defense and industry. In defense they observe a relatively small number of complex and expensive models. The complexity is related to the fact that there is much interaction between the constituting parts of the models, much more than in industrial models. Military models are often running on the edge of what is possible. This mandates full control over the environment explaining the choice of general purpose languages. Due to the complexity extensive requirement analysis is customary which leads to high credibility of the models. This, and the high building costs involved makes full model reuse feasible. Although the experts observe problems here, mainly related to semantic interoperability, 
reimplementation is not an issue. HLA is experienced as a good tool solving many low level problems.

In industry HLA designers see many models being built of a similar kind. The most important issue here is cost which has to be kept low. These are the two main reasons why the standard approach in industry is to use COTS packages. They perceive industry as less focused on distributed simulation which explains their observation that COTS and HLA hardly go together. An additional argument is that HLA is developed for the military and that typical industry related aspects have not been taken into account when designing this standard. Subsetting HLA towards the functionality needed by industry might be a good approach. In the opinion of the HLA developers, adopting HLA into COTS models is not a task for the model builder but for the package developers.

Another difference between the two application domains is that in defense there is one big player, the US DoD. This explains that in many cases, but not all, information hiding is not a big issue. In industry the experts see many players who are reluctant to cooperate out of fear to lose customers, which hampers development into the direction of coupling models written in different packages.

The experts involved in designing HLA envision a future in which ideas related to the use of Internet are implemented, repositories offering services that a customer may use, and lightweight clients. This entails a new business model in which clients are charged for services instead of packages.

\subsection{Simulation Practitioners in Defense}

The defense simulation practitioners mention that they see simulation modeling on a larger scale in defense than in industry, and that military simulation modeling is more research like, which entails that more control is needed over tools and the environment. This explains why defense uses general purpose languages. They also observe more concern in the military domain on security aspects which leads to more emphasis on validation.

They agree that reuse is important, but again the semantic interoperability problem is emphasized. Submodels are often not designed with networking in mind with the effect that the data models do not fit together. This leads to the need to update the FOM of models in the case of applying HLA, and to change their internal workings. Sometimes one has to start anew from scratch, and it can even be the case that there is not enough time, budget or expertise to combine the submodels at all.

Concerning information hiding the defense practitioners state that it was a big issue in their projects. The experts from defense perceive complexity, lack of transparency and insufficient performance as the most severe drawbacks of HLA. The complexity cannot be avoided be- cause distributed simulation in itself is complex. They define transparency similar to the HLA vendors as the possibility to access the levels below the HLA RTI. Insufficient performance led one of the experts to implement a new RTI. Hierarchical federations and the option to specify more elaborate data models were mentioned as useful additions to the HLA standard. They perceive as the biggest advantage of HLA that it is a standard.

Experts from defense propose an idealized architecture that hides as much complexity as possible from the novice user offering only basic functionality, viz. data exchange, time management, and execution control. Further, experienced users should be allowed more functionality and access to deeper layers enabling him to change default settings.

\subsection{Commercial HLA Vendors}

Although the commercial HLA vendors are open for collaboration with industry, they are squarely targeting the defense domain. The main reason is that they hardly observe HLA projects in industry whereas, due to the US DoD mandate, defense has adopted HLA. The HLA vendors observe that COTS vendors are reluctant to cooperate with each other. They can understand this because they see no added value for them in agreeing on a common standard. Instead, they observe the tendency that COTS vendors try to lock their customers to their own package.

Concerning information hiding, a nice example is given by one of the experts, viz., simulation based acquisition. Both experts stated that reuse is common in defense. They also mentioned the difficulty of semantically aligning the models to be combined. Not surprisingly, they see HLA as a good tool, that hides low level technical details, is general and not locked to one specific area. As drawbacks of HLA they mention the steep learning curve and the need for users who are not only good programmers, but high level thinkers as well. As regards performance they admit that the DMSO RTI is not efficient enough, and that their products try to remedy this. Features that they would like to see added to the HLA standard are extendibility, fault tolerance, better security, the possibility to cooperate between different RTI implementations and better transparency in the sense that lower layers should remain visible through HLA.

In order to obtain greater acceptance of distributed simulation in industry HLA vendors mention middleware and the need for a protocol on the wire level which would enable package builders to write their own implementation rather than relying on RTI implementations built by others. Similar to the HLA developers, one expert envisages a new business model in which the modeler would combine parts obtained from the net which then would be put to work using grid computing. 


\section{CATEGORIZATION OF THE COLLECTED DATA}

This section organizes the data from Section 3 in a structured framework through coding. Coding involves extracting from the data obtained the relevant topics that were raised, and dividing these into subtopics. The resulting list forms the basis of a "theory" that we present in a separate paper (Boer, et al. 2006b).

As a starting point for our categorization we consider the list on which our interview was based, cf. Section 2.2. We will use the items from this list as a preliminary list of topics. An analysis of the answers that we obtained for these parts will generate a list of subtopics, one for each part. During this categorization process we will observe that some subtopics are reconsidered in the answers in other parts as well. This will lead to a regrouping of the subtopics in our final list, and also to the introduction of new topics. At the end of this section we will present the final list in a succinct form.

Part 1 of our interview was included to get an idea of the experience of the interviewed experts. This part was intended for validation purposes and the answers did not yield insights in the research questions we are studying here.

Part 2 focused on the difference between simulation in defense and in industry. We obtained several categories of answers. One category was related to the difference in the business model of defense and industry. This influences the goals of the models built in the various domains, and the experts observed differences in the structure and type of the applications. Also differences in software engineering aspects were mentioned. The experts also pointed at similarities, in the sense that in industry models are built with the typical attributes of the ones in defense and vice versa.

Part 3 dealt with reuse. Three subtopics emerge here. First of all there is the issue of full model reuse versus reusing parts of a model. Secondly, there was a discussion on a rather high level, viz. the level of distributed simulation in general. Issues here are aspects related to the complexity of the models, and aspects related to the trust in the models, which are influenced by software engineering issues around specification, verification and validation, cf. some of the answers in Part 2. The third subtopic deals with the problems encountered. There are low level technical problems (partly) solved by the HLA standard, and there is the big mainly unsolved problem of semantic interoperability.

Part 4 discussed information hiding. The answers in this part can be categorized into two subtopics, we obtained examples of information hiding, both in defense and in industry, and we observed that the opinions diverged regarding the extent to which information hiding is an issue in current simulation projects.
Part 5 was aimed at the difficulties and the benefits of applying the HLA standard. Benefits could be subdivided into three categories: HLA hides low level details, HLA is a standard, and HLA offers a general solution not tied to one specific application area. As regards the difficulties we encountered two types of comments here. The first type is purely HLA related, the complexity of HLA, efficiency issues and the lack of transparency of HLA. The second type has to do with answers given from a higher level viewpoint, namely distributed simulation in general. Notice that we observed the same subdivision when discussing Part 3. Issues on this higher level are the low level knowledge and the knowledge on general systems needed from the practitioners, and issues around cost and benefit. A final group of comments triggered in Part 5 focused on desirable additions to the HLA standard.

Part 6 discussed integration of HLA and COTS. Our impression that this hardly occurs in industrial projects was confirmed by almost all experts we interviewed. The first subtopic deals with the reasons behind this. We obtained remarks related to the fact that HLA is applied mainly in defense, to the fact that HLA uses notions that are not available in COTS packages, viz. ownership. Further there were answers focusing on the inaccessibility of the lower levels of COTS packages, e.g., the simulation engine, on the fact that HLA is big, and on the difference between the high level COTS interface versus the low level HLA RTI calls. The second subtopic we observe in the answers in Part 6 deals with the pros and cons of middleware and adaptors. These issues were again raised in the answers in Part 9.

Part 7 was only used with the COTS vendors. It dealt with the advantages and disadvantages of combining COTS models. We derive two subtopics here, one consisting of the opportunities in industry (collaborative design and development, heterogeneity, emulation). The other subtopic was already identified when discussing Part 3, viz. the differences in the business model of industry and defense.

Part 8 was introduced to obtain information on support for HLA for industry. This part was only addressed at HLA vendors. The outcome here was univocal; the vendors only address the defense domain.

The last part was on an "idealized" architecture for industrial distributed simulation. We observe the following subtopics here. First of all there is the idea of a solution applying Web service like notions leading to a new business model. Secondly there were answers discussing the feasibility of adaptors and middleware. Another subtopic centered around industry needing only part of the functionality that defense applies. Here two approaches surfaced either define a subset of HLA to work with, or introduce a new lightweight standard with restricted functionality. The final subtopic focuses on the need for a high level COTSlike interface for this new architecture. 
The above considerations are captured in Table 1. Notice that some regrouping has taken place. One new topic has been introduced in the list, characteristics of projects favoring a distributed simulation approach. Under this new topic some old ones have been grouped. Furthermore subtopics which occurred in the above discussion more than once have been put in one place.

Table 1: Topics Derived from the Survey

\begin{tabular}{|c|c|}
\hline Topics & Subtopics \\
\hline $\begin{array}{l}\text { The difference between de- } \\
\text { fense and industry in applying } \\
\text { distributed simulation }\end{array}$ & $\begin{array}{l}\text { - Difference in the business model } \\
\text { - Difference in the goal of the models built } \\
\text { - Difference in the type and structure of the models built } \\
\text { - Difference in software engineering aspects } \\
\text { - Specification } \\
\text { - Verification } \\
\text { - Validation }\end{array}$ \\
\hline $\begin{array}{l}\text { Characteristics of simulation } \\
\text { projects making a distributed } \\
\text { approach feasible }\end{array}$ & $\begin{array}{l}\text { - Reuse } \\
\text { - Full model reuse vs. partial reuse } \\
\text { - Arguments on the level of distributed simulation } \\
\text { - Complexity of the models } \\
\text { - Trust in the models } \\
\text { - The extent to which information hiding plays a role } \\
\text { - Ther characteristics (collaboration, heterogeneity, emulation) }\end{array}$ \\
\hline $\begin{array}{l}\text { Difficulties and benefits of } \\
\text { applying HLA }\end{array}$ & 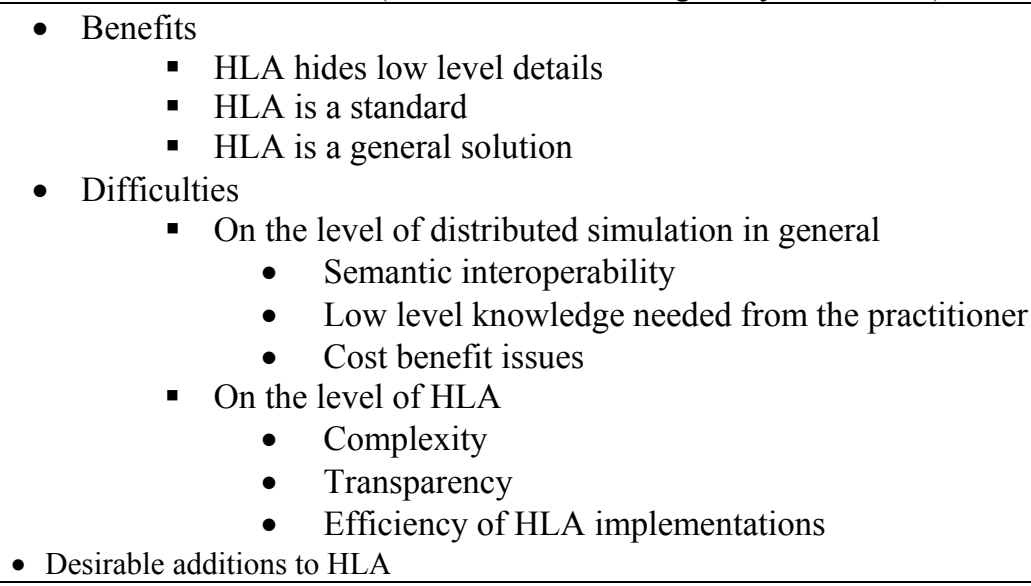 \\
\hline $\begin{array}{l}\text { Integrating HLA and COTS } \\
\text { (HLA hardly applied in COTS } \\
\text { related projects) }\end{array}$ & $\begin{array}{l}\text { - HLA mainly applied in defense } \\
\text { - HLA notions not covered in COTS packages } \\
\text { - Closed feature of COTS packages } \\
\text { - HLA is too big } \\
\text { - High level COTS interface vs. low level HLA RTI calls }\end{array}$ \\
\hline "Idealized" architecture & $\begin{array}{l}\text { - } \text { Ideas from the Web } \\
\text { - Feasibility of adaptors and middleware } \\
\text { - Industry needs only restricted functionality } \\
\text { - Subsetting HLA } \\
\text { - A new lightweight architecture } \\
\text { - High level COTS like interface }\end{array}$ \\
\hline
\end{tabular}




\section{CONCLUSIONS AND RELATED WORK}

We have conducted an extensive survey the results of which are summarized in the previous section. These results can be organized along the lines of Table 1 . We have concluded that the HLA standard is rarely used for integrating simulation models designed and developed in COTS simulation packages. Further, we highlighted several reasons for this, reasons relating to distributed simulation in general, to the HLA standard itself, and to the relation between the COTS simulation packages and the HLA standard. Interestingly all three categories connect to the very general economic reason of the cost benefit ratio.

Based on these reasons, our next goal is to answer the following question:

\section{How can we make distributed simulation and existing distributed solutions, like HLA, more attractive to the industrial community?}

In order to realize this there is a need for a COTS based distributed simulation architecture which might improve the perceptible cost benefit ratio. This idea has been worked out in the paper (Boer, et al. 2006b), where a requirement list for an architecture for industrial distributed simulation is proposed based on the results of the survey described here.

\section{ACKNOWLEDGMENTS}

We would like to thank the simulation experts for the useful discussions during the interview survey and to Erasmus University Rotterdam for supporting this research.

\section{REFERENCES}

Boer, C. A. 2005. Distributed Simulation in Industry Ph.D. Thesis, Erasmus University Rotterdam, The Netherlands. Available via <https://ep.eur.nl/ handle/1765/6925 > [accessed June 22, 2006]

Boer, C. A., A. de Bruin, and A. Verbraeck. 2006a. Distributed Simulation in Industry - A Survey, Part 1 The COTS Vendors, Accepted in Proceedings of the 2006 Winter Simulation Conference, eds. L. F. Perrone, F. P. Wieland, J. Liu, B. G. Lawson, D. M. Nicol, and R. M. Fujimoto. Piscataway, New Jersey: Institute of Electrical and Electronics Engineers.

Boer, C. A., A. de Bruin, and A. Verbraeck. 2006b. The use of Distributed Simulation in Industry - A Survey, Submitted to Simulation: Transactions of The Society for Modeling and Simulation International.

Creswell, J. W. 2003. Research Design. Qualitative, Quantitative, and Mixed Methods Approaches. (Second ed.), Thousand Oaks, California, USA. SAGE Publications.
Linstone, H. A. 1978. The Delphi Technique, in Handbook of Future Research, ed. J. Fowles, London: Greenwood Press, pp. 273-300.

Linstone, H. A. and M. Turoff. 1975. The Delphi Method. Techniques and Applications., London, UK: AddisonWesley.

Schreiber, G., H. Akkermans, A. Ajewierden, R. De Hoog, N. Shadbolt, W. Van de Velde, and B. Wielinga. 2000. Knowledge Engineering and Management, Cambridge, Massachusetts, USA: MIT Press.

Seale, C., G. Gobo, J. F. Gubrium, and D. Silverman. 2004. Qualitative Research Practice, London, UK. SAGE Publications Ltd.

Turban, E. 1995. Decision Support and Expert Systems, Englewood Cliffs, New Jersey, USA: Prentice-Hall.

\section{AUTHOR BIOGRAPHIES}

CSABA ATTILA BOER is a product manager at TBA $\mathrm{BV}$, one of the leading logistics and simulation consultancy firms in Europe. He holds a Ph.D. in Computer Science and Logistics from Erasmus University Rotterdam. His research interests include distributed simulation, distributed virtual environments, port logistics, and port simulation and emulation. His e-mail address is <csaba.boer@tba.nl>

ARIE DE BRUIN is a full professor at Delft University of Technology. He got his Ph.D. on semantics of programming languages and since then he has specialized in investigating implementation issues from a theoretician's point of view. One of his current research interest is distributed simulation, most notably the essential concepts thereof, and the soundness of the implementation of these concepts. His e-mail address is <a.debruin@tudelft.nl>

ALEXANDER VERBRAECK is an associate professor in the Systems Engineering Group of the Faculty of Technology, Policy and Management of Delft University of Technology, and a part-time full professor in supply chain management at the R. H. Smith School of Business of the University of Maryland. He is a specialist in discrete-event simulation for real-time control of complex transportation systems and for modeling business systems. His current research focus is on the development of open and generic libraries of object oriented simulation building blocks in the Java programming language. His e-mail address is <alexandv@tbm.tudelft.nl> 